Carta

al editor

REV EXP MED

2019;5(4).

Octubre - Diciembre

\title{
Bioimpresión, una alternativa de la medicina
}

\author{
Bioprinting, an alternative medicine
}

Jhon Abner Herrera-Vasquez 1,a, Mileny Jeraldin Silva-Nuñez 1,a, Zhandra Lizette Arce-Gil 1,b

Sr. Editor:

En los últimos años, la impresión tridimensional (3D) ha ido ganando terreno por su versatilidad y aplicaciones en diversos ámbitos, en especial en el área de la salud, por lo que resulta de interés su aplicación para la creación de modelos anatómicos, prototipos, prótesis personalizadas, entre muchas otras aplicaciones médicas. Esta técnica fue desarrollada originalmente para imprimir capas secuenciales finas de material, seguido de un proceso de curado con luz ultravioleta para formar sólidas estructuras tridimensionales al que se denominó "estereolitografía", descrita por primera vez en 1986 por Charles W. Hull que con el transcurso de los años, esta técnica ha evolucionado a pasos agigantados y se ha logrado volverla más provechosa y compleja ${ }^{(1,2)}$.

Actualmente en el campo quirúrgico se ha ido empleando como una herramienta para mejorar cualquier anomalía compleja, como en cirugía de columna, de abdomen, ortopédica, cardiovascular y neurocirugía; donde se simulan todos los pasos quirúrgicos complicados por adelantado. Usando modelos prototipo puede ayudar a prever complicaciones intra y postoperatorias dando una mejora significativa en el diagnóstico y tratamiento debido a la mejor apreciación y precisión de la estructura afectada ${ }^{(3)}$.

En adición, con las técnicas de bioimpresión 3D los científicos han logrado crear células de hígado que son capaces de vivir durante más de 40 días, también se ha logrado la impresión de láminas de tejido cardíaco y células madre que pueden reproducir diferentes tipos de tejidos humanos. La bioimpresión 3D de un prototipo sugiere que pronto estaremos en condiciones de reemplazar todo, desde una oreja hasta un corazón entero, de hecho el Instituto Wake Forest de Medicina Regenerativa imprime estructuras de oreja, nariz y hueso que pueden ser luego recubiertos con células para crear estas partes del cuerpo, lo cual está siendo aplicada en implantes personalizados como prótesis de rodilla y cadera "hechos a la medida", para posteriormente hacer una reconstrucción de dichas estructuras ${ }^{(4,5)}$.

Como futuros profesionales de la salud queremos resaltar los avances de la bioimpresión 3D los cuales pueden ser implementados en las universidades con fines educativos, es decir, en los cursos básicos como anatomía, histología, y cursos de mayor complejidad como cirugía y ginecobstetricia. Así permitirá mejorar el desempeño y adiestramiento del estudiante, así mismo resaltar que en un futuro próximo por medio de la bioimpresión 3D también se logre salvar muchas vidas con diferentes enfermedades donde se pueda realizar la implantación de tejidos u órganos más complejos, de la manera más rápida, con un bajo costo y sin perder tiempo vital para el paciente. Por último, se logre superar los límites de dimensiones de la bioimpresión. ¿Llegará el día que se logre bio imprimir un cuerpo humano entero? ¿La bio impresión 3D podrá ser una alternativa terapéutica para la regeneración celular?...

\footnotetext{
1. Facultad de Medicina, Universidad Santo Toribio de Mogrovejo, Chiclayo, Perú.

a. Estudiante de Medicina Humana.

b Biólogo Microbiólogo, Magíster en Microbiología.
}

Correspondencia: Jhon Abner Herrera Vasquez. 
Fuentes de financiamiento: autofinanciado.

Conflictos de interés: Los autores declaran no tener conflictos de interés.

\section{REFERENCIAS BIBLIOGRÁFICAS}

1. Takagishi K, Umezu S. Development of the Improving Process for the 3D Printed Structure. Sci Rep. 2017; 7(1):1-10.

2. García-Valadez LR, Espinoza-Gutiérrez A, Rivas-Montero JA, Hernández-Méndez-Villamil E, Santiago-García A, Banegas-Ruiz R, et al. Impresión de modelos $3 \mathrm{D}$ para fracturas de radio distal: un estudio piloto en el Instituto Nacional de Rehabilitación. Rev Sanid Milit Mex. 2017; 71(4):36673.

3. Martínez G, Estrada F. Hacia la medicina del 2030. UCE Ciencia Rev postgrado. 2019; 7(1):1-9.

4. César-Juárez AA, Olivos-Meza A, Landa-Solís C, Cárdenas-Soria VH, Silva-Bermúdez PS, Suárez-Ahedo C, Olivos-Díaz B, Ibarra-Ponce de León JC. Uso y aplicación de la tecnología de impresión y bioimpresión 3D en medicina. Use Appl 3D Print bioimpression Technol Med. 2018; 61(6):4351.

5. Gil A. Las impresoras 3D como herramientas científicas. Obs Astron la Univ Val. 2019; 61(21):1-8.

Revisión de pares: Recibido: 28/11/2019 Aceptado: 27/12/ 2019 